\title{
A pair of oscillators interacting with a common heat bath
}

\author{
G. W. Ford \\ Department of Physics, University of Michigan, Ann Arbor, MI 48109-1040 USA \\ R. F. O'Connell \\ Department of Physics and Astronomy, \\ Louisiana State University, Baton Rouge, LA 70803-4001 USA
}

(Dated: March 12, 2018)

\begin{abstract}
Here the problem considered is that of a pair of oscillators coupled to a common heat bath. Many, if not most, discussions of a single operator coupled to a bath have used the independent oscillator model of the bath. However, that model has no notion of separation, so the question of phenomena when the oscillators are near one another compared with when they are widely separated cannot be addressed. Here the Lamb model of an oscillator attached to a stretched string is generalized to illustrate some of these questions. The coupled Langevin equations for a pair of oscillators attached to the string at different points are derived and their limits for large and small separations obtained. Finally, as an illustration of a different phenomenon, the fluctuation force between a pair of masses attached to the string is calculated, with closed form expressions for the force at small and large separations..

PACS numbers: 05.40.-a, 03.65.Yz, 03.65.-w
\end{abstract}


Many, if not most, discussions of a quantum oscillator interacting with a quantum heat bath have been based on the independent oscillator mode of the bath. A major advantage of this model is that it can be shown to be equivalent with the most general linear passive bath, even one coupled to multiple degrees of freedom. [1, 2] However, an attempt to construct such a model to describe two separated oscillators does not lead to results of interest, essentially because the independent oscillator model does not lend itself to the notion of physical separation. However, the Lamb model of an oscillator attached to a stretched string does have the notion of position: a pair of oscillators can be attached to separated points on the string. This model was introduced by Horace Lamb in 1900 [3], in order to better understand radiation reaction in electrodynamics. Later it was shown by Lewis and Thomas [4] that the Lamb model is a special case of the independent oscillator model. Thus we have an independent oscillator model (the oscillators are the normal mode oscillators of the string) in which the notion of separation is obvious. Our aim in this paper will be to describe some of the interesting physics that arises when a pair of oscillators are attached to separated points on the string.

We begin by defining the model and using it to obtain a pair of coupled quantum Langevin equations describing the motion of the pair of oscillators. The procedure here is a straightforward generalization of that used to obtain the equation for a single oscillator attached to the string.[1] The results, however, show some striking phenomena: interaction of the oscillators produced by waves in the string propagating from one to the other, correlation of the random forces acting on each oscillator. We then show how simple descriptions of the motion arise in the limits of large and small separations.

As an example of a different phenomenon, we then turn to a calculation of the fluctuation force acting between a pair of masses attached to the string. This force is a one dimensional analog of the London-van der Waals force between molecules or the Casimir force between parallel plates. As in those cases, we find simple expressions for the force at small and large separations.

The Lamb model for a pair of oscillators coupled to points on a stretched string is described by the Lagrangian:

$$
L=\frac{1}{2} m_{1} \dot{x}_{1}^{2}-\frac{1}{2} K_{1} x_{1}^{2}+\frac{1}{2} m_{2} \dot{x}_{2}^{2}-\frac{1}{2} K_{2} x_{2}^{2}+\int_{-\infty}^{\infty} d y\left[\frac{\sigma}{2}\left(\frac{\partial u}{\partial t}\right)^{2}-\frac{\tau}{2}\left(\frac{\partial u}{\partial y}\right)^{2}\right],
$$

where $u(y)$ is the string displacement. The mass per unit length of the string is $\sigma$ and the 
tension is $\tau$. Note that the string is stretched along the $y$-axis and the oscillator displacements are along the $x$-axis, perpendicular to the string. Note also that there is no interaction term in the Lagrangian; instead for a pair of oscillators one imposes the constraints:

$$
x_{1}(t)=u\left(y_{1}, t\right), \quad x_{2}(t)=u\left(y_{2}, t\right)
$$

where $y_{1}$ and $y_{2}$ are the positions of the oscillators along the string. The equations of motion of the oscillators are

$$
\begin{aligned}
& m_{1} \ddot{x}_{1}+K_{1} x_{1}=f_{1}(t), \\
& m_{2} \ddot{x}_{2}+K_{2} x_{2}=f_{2}(t),
\end{aligned}
$$

where $f_{1}(t)$ and $f_{2}(t)$ are the constraint forces exerted by the string on the individual oscillators. The field equation of motion for the string becomes the inhomogeneous wave equation:

$$
\frac{\partial^{2} u}{\partial t^{2}}-c^{2} \frac{\partial^{2} u}{\partial y^{2}}=-\frac{f_{1}(t)}{\sigma} \delta\left(y-y_{1}\right)-\frac{f_{2}(t)}{\sigma} \delta\left(y-y_{2}\right)
$$

where $c=\sqrt{\tau / \sigma}$ is the wave velocity. The retarded solution of this equation is

$$
u(y, t)=u^{\mathrm{h}}(y, t)-\frac{1}{\zeta} \int_{-\infty}^{t-\left|y-y_{1}\right| / c} d t^{\prime} f_{1}\left(t^{\prime}\right)-\frac{1}{\zeta} \int_{-\infty}^{t-\left|y-y_{2}\right| / c} d t^{\prime} f_{2}\left(t^{\prime}\right),
$$

where $u^{\mathrm{h}}(y, t)$ is the operator solution of the homogeneous equation corresponding to the free motion of the string in the absence of the oscillators and

$$
\zeta=2 \sigma c=2 \sqrt{\sigma \tau}
$$

is the friction constant. Differentiating with respect to $t$, we find

$$
\frac{\partial u(y, t)}{\partial t}=\frac{\partial u^{\mathrm{h}}(y, t)}{\partial t}-\frac{1}{\zeta} f_{1}\left(t-\frac{\left|y-y_{1}\right|}{c}\right)-\frac{1}{\zeta} f_{2}\left(t-\frac{\left|y-y_{2}\right|}{c}\right) .
$$

Putting first $y=y_{1}$ and then $y=y_{2}$, we find using the constraints (2) that we can write

$$
\begin{aligned}
& f_{1}(t)+f_{2}\left(t_{\mathrm{R}}\right)=-\zeta \dot{x}_{1}(t)+F_{1}(t), \\
& f_{1}\left(t_{\mathrm{R}}\right)+f_{2}(t)=-\zeta \dot{x}_{2}(t)+F_{2}(t),
\end{aligned}
$$

where $t_{\mathrm{R}}$ is the retarded time,

$$
t_{\mathrm{R}}=t-\frac{\left|y_{1}-y_{2}\right|}{c}
$$


while $F_{1}(t)$ and $F_{2}(t)$ are fluctuating forces:

$$
F_{1}(t)=\zeta \frac{\partial u^{\mathrm{h}}\left(y_{1}, t\right)}{\partial t}, \quad F_{2}(t)=\zeta \frac{\partial u^{\mathrm{h}}\left(y_{2}, t\right)}{\partial t}
$$

We can solve the equations (8) to find explicit expressions for $f_{1}(t)$ and $f_{2}(t)$, but it is simpler to form the Fourier transforms to get (remember $t_{\mathrm{R}}$ is given by (9)

$$
\left(\begin{array}{cc}
1 & e^{i \omega\left|y_{1}-y_{2}\right| / c} \\
e^{i \omega\left|y_{1}-y_{2}\right| / c} & 1
\end{array}\right)\left(\begin{array}{c}
\tilde{f}_{1}(\omega) \\
\tilde{f}_{2}(\omega)
\end{array}\right)=i \omega \zeta\left(\begin{array}{c}
\tilde{x}_{1}(\omega) \\
\tilde{x}_{2}(\omega)
\end{array}\right)+\left(\begin{array}{c}
\tilde{F}_{1}(\omega) \\
\tilde{F}_{2}(\omega)
\end{array}\right) .
$$

If we form the Fourier transform of the equations of motion (3) we get

$$
\left(\begin{array}{cc}
-m_{1} \omega^{2}+K_{1} & 0 \\
0 & -m_{2} \omega^{2}+K_{2}
\end{array}\right)\left(\begin{array}{c}
\tilde{x}_{1}(\omega) \\
\tilde{x}_{2}(\omega)
\end{array}\right)=\left(\begin{array}{c}
\tilde{f}_{1}(\omega) \\
\tilde{f}_{2}(\omega)
\end{array}\right)
$$

Eliminating the Fourier transform of the constraint forces between these two equations, we can write

$$
\left(\begin{array}{cc}
-m_{1} \omega^{2}-i \omega \zeta+K_{1} & \left(-m_{2} \omega^{2}+K_{2}\right) e^{i \omega\left|y_{1}-y_{2}\right| / c} \\
\left(-m_{1} \omega^{2}+K_{1}\right) e^{i \omega\left|y_{1}-y_{2}\right| / c} & -m_{2} \omega^{2}-i \omega \zeta+K_{2}
\end{array}\right)\left(\begin{array}{c}
\tilde{x}_{1}(\omega) \\
\tilde{x}_{2}(\omega)
\end{array}\right)=\left(\begin{array}{c}
\tilde{F}_{1}(\omega) \\
\tilde{F}_{2}(\omega)
\end{array}\right) .
$$

The susceptibility matrix is therefore

$$
\alpha(\omega)=\left(\begin{array}{cc}
-m_{1} \omega^{2}-i \omega \zeta+K_{1} & \left(-m_{2} \omega^{2}+K_{2}\right) e^{i \omega\left|y_{1}-y_{2}\right| / c} \\
\left(-m_{1} \omega^{2}+K_{1}\right) e^{i \omega\left|y_{1}-y_{2}\right| / c} & -m_{2} \omega^{2}-i \omega \zeta+K_{2}
\end{array}\right)^{-1} .
$$

The quantum Langevin equations are

$$
\begin{aligned}
& m_{1} \ddot{x}_{1}(t)+\zeta \dot{x}_{1}(t)+K_{1} x_{1}(t)+m_{2} \ddot{x}_{2}\left(t_{\mathrm{R}}\right)+K_{2} x_{2}\left(t_{\mathrm{R}}\right)=F_{1}(t), \\
& m_{2} \ddot{x}_{2}(t)+\zeta \dot{x}_{2}(t)+K_{2} x_{2}(t)+m_{1} \ddot{x}_{1}\left(t_{\mathrm{R}}\right)+K_{1} x_{1}\left(t_{\mathrm{R}}\right)=F_{2}(t) .
\end{aligned}
$$

The form of these equations makes clear that, at time $t$, there is an interaction between $x_{1}=u\left(y_{1}\right)$ and $x_{2}=u\left(y_{2}\right)$. In particular, we note that when there is an effect at $y_{1}$ due to the heat bath that it does not effect $y_{2}$ until a later time $\left|y_{1}-y_{2}\right| / c$.

To get explicit expressions for the force correlations, we recall the well known expression for the operator displacement of a string of length $L:[5]$

$$
u^{\mathrm{h}}(y, t)=\sum_{k} \sqrt{\frac{\hbar}{2 \sigma L \omega}}\left(a_{k} e^{i(k y-\omega t)}+a_{k}^{\dagger} e^{-i(k y-\omega t)}\right),
$$


where $\omega=c|k|$ and $k=\frac{2 \pi n}{L}$ with $n=0, \pm 1, \pm 2, \cdots$. In equilibrium at temperature $T$,

$$
\left\langle a_{k} a_{k^{\prime}}^{\dagger}+a_{k}^{\dagger} a_{k^{\prime}}\right\rangle=\operatorname{coth} \frac{\hbar \omega}{2 k_{B} T} \delta_{k, k^{\prime}}
$$

With this in the expressions (10) for the fluctuating forces, we find (in the limit $L \rightarrow \infty$ ) the correlations:

$$
\begin{gathered}
\frac{1}{2}\left\langle F_{1}(t) F_{1}\left(t^{\prime}\right)+F_{1}\left(t^{\prime}\right) F_{1}(t)\right\rangle=\frac{1}{2}\left\langle F_{2}(t) F_{2}\left(t^{\prime}\right)+F_{2}\left(t^{\prime}\right) F_{2}(t)\right\rangle \\
=\frac{\hbar \zeta}{\pi} \int_{0}^{\infty} d \omega \omega \operatorname{coth} \frac{\hbar \omega}{2 k_{B} T} \cos \left[\omega\left(t-t^{\prime}\right)\right] \\
=\frac{\zeta k T}{2} \frac{d}{d t} \operatorname{coth} \frac{\pi k T\left(t-t^{\prime}\right)}{\hbar}, \\
\frac{1}{2}\left\langle F_{1}(t) F_{2}\left(t^{\prime}\right)+F_{2}\left(t^{\prime}\right) F_{1}(t)\right\rangle=\frac{\hbar \zeta}{\pi} \int_{0}^{\infty} d \omega \omega \operatorname{coth} \frac{\hbar \omega}{2 k_{B} T} \cos \left[\omega\left(t-t^{\prime}\right)\right] \cos \frac{\omega\left(y_{1}-y_{2}\right)}{c} \\
=\frac{\zeta k T}{4} \frac{d}{d t}\left(\operatorname{coth} \frac{\pi k T\left(t-t^{\prime}-\frac{y_{1}-y_{2}}{c}\right)}{\hbar}+\operatorname{coth} \frac{\pi k T\left(t-t^{\prime}+\frac{y_{1}-y_{2}}{c}\right)}{\hbar}\right) .
\end{gathered}
$$

Here we should note in the explicit expressions that $d \operatorname{coth}(x) / d x=2 \delta(x)-\operatorname{csch}^{2}(x)[6,7]$.

With these expressions for the correlations, we see from the Langevin equations (15) that, for large separations and times short compared with $\left|y_{1}-y_{2}\right| / c$, the retarded time (6) is in the distant past and the equations become decoupled. Each equation becomes a Langevin equation for a single oscillator interacting with the bath. There can still be correlation between the oscillators, for example they can be in an entangled state, but there are no bath-induced correlations.

The case of very small separations is more subtle. To simplify the discussion we assume the oscillators are identical, that is, $m_{1}=m_{2}=m$ and $K_{1}=K_{2}=K$. Then by adding and subtracting the two Langevin equations (15) and introducing the variables:

$$
X=\frac{x_{1}+x_{2}}{2}, \quad x=x_{1}-x_{2}
$$

we obtain the separate equations [8]

$$
\begin{aligned}
m \ddot{X}(t)+\zeta \dot{X}(t)+K X(t)+m \ddot{X}\left(t_{\mathrm{R}}\right)+K X\left(t_{\mathrm{R}}\right) & =\frac{F_{1}(t)+F_{2}(t)}{2}, \\
m \ddot{x}(t)+\zeta \dot{x}(t)+K x(t)-m \ddot{x}\left(t_{\mathrm{R}}\right)-K x\left(t_{\mathrm{R}}\right) & =F_{1}(t)-F_{2}(t) .
\end{aligned}
$$

If we set $\left|y_{1}-y_{2}\right|=0$, then $t_{\mathrm{R}}=t$. Moreover, for very small separations, it is important to note that that all three of the correlations ( 18) of the fluctuation forces are the same; there 
is only one fluctuation force. Therefore the first of the equations (20b) becomes exactly the Langevin equation for a single oscillator whose mass is the sum of the masses and with a spring constant equal to the sum of the spring constants. This is what we should expect, when the two oscillators merge they should become one. But what about the second of the equations (20b)? With $\left|y_{1}-y_{2}\right|=0$ this equation becomes

$$
\zeta \dot{x}(t)=0
$$

with no fluctuating force. Thus $x=x_{1}-x_{2}$ can take on any constant value!

To better understand this strange result we consider the free energy for the system, which is given by the remarkable formula: $[2,8]$

$$
F\left(\left|y_{1}-y_{2}\right|, T\right)=\frac{1}{\pi} \int_{0}^{\infty} d \omega f(\omega, T) \operatorname{Im}\left\{\frac{d \log \operatorname{det} \alpha\left(\omega+i 0^{+}\right)}{d \omega}\right\}
$$

where $\alpha$ is the polarizability matrix (14) and

$$
f(\omega, T)=k T \log \left(2 \sinh \frac{\hbar \omega}{2 k T}\right)
$$

is the free energy of a single oscillator of natural frequency $\omega$. I now we put $\left|y_{1}-y_{2}\right|=0$ in the polarizability matrix (14) we find

$$
\operatorname{det} \alpha(\omega)=\frac{1}{-i \omega \zeta} \times \frac{1}{-\left(m_{1}+m_{2}\right) \omega^{2}+K_{1}+K_{2}-i \omega \zeta} .
$$

Here the second factor is exactly the polarizability of a single oscillator with mass the sum of the masses and spring constant equal to the sum of the spring constants. When put in the remarkable formula this factor will give the corresponding free energy. The first factor will give rise to an additional free energy

$$
\Delta F(0, T)=\frac{1}{2} f(0, T)
$$

where we have used the well known identity: $\operatorname{Im}\left\{1 /\left(\omega+i 0^{+}\right)\right\}=-\pi \delta(\omega)$ and the factor of $\frac{1}{2}$ takes into account the fact that the delta function is at the initial point of the integration. This additional free energy is infinite: an oscillator whose natural frequency is zero is an unbounded free particle. We conclude that, in the limit $\left|y_{1}-y_{2}\right| \rightarrow 0$, the free energy of the system is infinite, but that the divergent part can be isolated and remainder is finite and can be recognized as the free energy of a single oscillator coupled to the bath. 
We now turn to a calculation of the fluctuation force between two masses attached to a string. In order to calculate the stress between the two masses, we must first obtain the solution of the equation of motion of the string $u(y, t)$ corresponding to an external force $f(y, t)$, in the form of an integral involving the Green function $G\left(y, y^{\prime} ; t\right)$. We then solve for the Fourier transform of the Green function at points between the two masses, which will involve reflection coefficients $r_{1}$ at $y_{1}$ and $r_{2}$ at $y_{2}$. Next, the stress at a point between the two masses is calculated in terms of space and time derivatives of the solution of the equations of motion, which leads to an expression in terms of the spatial correlation function, which in turn is evaluated by means of the fluctuation-dissipation theorem in terms of the Green function.

Thus, we start with two equal masses $m$ attached to the string, one at $y_{1}$ the other at $y_{2}$. The Green function is used to express the solution of the equation of motion of the string corresponding to an external force $f(y, t)$ in the form

$$
u(y, t)=\int_{-\infty}^{t} d t^{\prime} \int_{-\infty}^{\infty} d y^{\prime} G\left(y, y^{\prime}: t-t^{\prime}\right) f\left(y,{ }^{\prime}, t^{\prime}\right) .
$$

Clearly the Green function $G\left(y, y^{\prime} ; t\right)$ that vanishes for negative times corresponds to an external force $\delta\left(y-y^{\prime}\right) \delta(t)$. For a source point $y^{\prime}$ and field point $y$ both in the interval between the two masses, the Fourier transform of the Green function is a solution of the inhomogeneous Helmholtz equation:

$$
\frac{\partial^{2} \tilde{G}\left(y, y^{\prime} ; \omega\right)}{\partial y^{2}}+\frac{\omega^{2}}{c^{2}} \tilde{G}\left(y, y^{\prime} ; \omega\right)=-\frac{1}{\tau} \delta\left(y-y^{\prime}\right) .
$$

Still in the region between the pair of masses $\left(y_{2}<y, y^{\prime}<y_{1}\right)$ the Green function for $y>y^{\prime}$ has the form of a plane wave reflecting off the mass at $y_{1}$, while for $y<y^{\prime}$ it has the form of a plane wave reflecting off the mass at $y_{2}$. In addition to satisfy the inhomogeneous equation, $\tilde{G}\left(y, y^{\prime} ; \omega\right)$ must be continuous and have a jump of $-1 / \tau$ in its derivative at $y=y^{\prime}$. It follows that

$$
\begin{aligned}
& \quad \tilde{G}\left(y, y^{\prime} ; \omega\right)= \\
& =\frac{i}{2 k \tau} \begin{cases}\frac{\left[e^{i k\left(y_{2}-y^{\prime}\right)}+r_{2} e^{-i k\left(y_{2}-y^{\prime}\right)}\right]\left[e^{i k\left(y-y_{1}\right)}+r_{1} e^{-i k\left(y-y_{1}\right)}\right]}{e^{-i k\left(y_{1}-y_{2}\right)}-r_{1} r_{2} e^{i k\left(y_{1}-y_{2}\right)}}, & y^{\prime}<y<y_{1} \\
\frac{\left[e^{i k\left(y^{\prime}-y_{1}\right)}+r_{1} e^{-i k\left(y^{\prime}-y_{1}\right)}\right]\left[e^{i k\left(y_{2}-y\right)}+r_{2} e^{-i k\left(y_{2}-y\right)}\right]}{e^{-i k\left(y_{1}-y_{2}\right)}-r_{1} r_{2} e^{i k\left(y_{1}-y_{2}\right)}}, & y_{2}<y<y^{\prime}\end{cases}
\end{aligned}
$$

where $r_{1}$ and $r_{2}$ are the reflection coefficients at $y_{1}$ and $y_{2}$, respectively. Note that the Green function is symmetric under interchange of $y$ and $y^{\prime}: \tilde{G}\left(y^{\prime}, y ; \omega\right)=\tilde{G}\left(y, y^{\prime} ; \omega\right)$. 
Next, we note that the mean of the stress $T$ at a point between the masses is equal to the mean of the energy density:

$$
\begin{aligned}
\langle T\rangle & =\left\langle\frac{1}{2} \sigma\left(\frac{\partial u}{\partial t}\right)^{2}+\frac{1}{2} \tau\left(\frac{\partial u}{\partial y}\right)^{2}\right\rangle \\
& =\left[\left(\frac{1}{2} \sigma \frac{\partial^{2}}{\partial t \partial t^{\prime}}+\frac{1}{2} \tau \frac{\partial^{2}}{\partial y \partial y^{\prime}}\right) C\left(y, y^{\prime} ; t-t^{\prime}\right)\right]_{\substack{t^{\prime}=t \\
y^{\prime}=y}},
\end{aligned}
$$

where

$$
C\left(y, y^{\prime} ; t-t^{\prime}\right)=\frac{1}{2}\left\langle u(y, t) u\left(y^{\prime}, t^{\prime}\right)+u\left(y^{\prime}, t^{\prime}\right) y(x, t)\right\rangle
$$

is the correlation function of the string displacement (only $t-t^{\prime}$ appears because of time translation invariance). The fluctuation-dissipation theorem relates this correlation function to the Green function:

$$
\tilde{C}\left(y, y^{\prime} ; \omega\right)=\frac{\hbar}{2 i} \operatorname{coth} \frac{\hbar \omega}{2 k_{B} T}\left[\tilde{G}\left(y, y^{\prime} ; \omega\right)-\tilde{G}\left(y^{\prime}, y ; \omega\right)^{*}\right]
$$

Therefore

$$
\langle T\rangle=\frac{\hbar}{2 \pi} \int_{-\infty}^{\infty} d \omega \operatorname{coth} \frac{\hbar \omega}{2 k_{B} T}\left[\left(\frac{1}{2} \sigma \omega^{2}+\frac{1}{2} \tau \frac{\partial^{2}}{\partial y \partial y^{\prime}}\right) \operatorname{Im}\left\{\tilde{G}\left(y, y^{\prime} ; \omega\right)\right\}\right]_{y^{\prime}=y} .
$$

Thus, using equation (28) for the Green function we get

$$
\langle T\rangle=\frac{\hbar}{4 \pi c} \int_{-\infty}^{\infty} d \omega \omega \operatorname{coth} \frac{\hbar \omega}{2 k_{B} T} \operatorname{Re}\left\{\frac{1+r_{1} r_{2} e^{2 i k\left|y_{1}-y_{2}\right|}}{1-r_{1} r_{2} e^{2 i k\left|y_{1}-y_{2}\right|}}\right\} .
$$

We interpret the fluctuation force between the masses as the difference between the stress in the presence of the attached masses and the free space stress $\left(r_{1}=r_{2}=0\right)$. That is,

$$
\begin{aligned}
F & =\langle T\rangle-\langle T\rangle_{0} \\
& =\frac{\hbar}{2 \pi c} \int_{-\infty}^{\infty} d \omega \omega \operatorname{coth} \frac{\hbar \omega}{2 k_{B} T} \operatorname{Re}\left\{\frac{r_{1} r_{2} e^{2 i k\left|y_{1}-y_{2}\right|}}{1-r_{1} r_{2} e^{2 i k\left|y_{1}-y_{2}\right|}}\right\}
\end{aligned}
$$

To proceed we must determine the reflection coefficients. To do so we consider scattering by a single mass at $y=0$, for which the scattering wave is of the form.

$$
u(y, t)=e^{-i \omega t}\left\{\begin{array}{c}
e^{i k y}+r e^{-i k y}, \quad y<0 \\
t e^{i k y}, \quad y>0
\end{array}\right.
$$

where $r$ and $t$ are the reflection and transmission coefficients, while $k=\omega / c$. At $y=0$ we must require continuity $([u]=0)$ and a jump in the derivative corresponding to the 
force exerted by the mass $\left([\tau \partial u / \partial y]=-m \omega^{2} u\right)$ The result is a pair of equations for the determination of $r$ and $t$. Their solution is

$$
t=\frac{\zeta}{\zeta-i m \omega}, \quad r=\frac{i m \omega}{\zeta-i m \omega},
$$

where $\zeta$ is given in Eq. (18). Therefore, since the masses are assumed identical, in the expression (34) for the fluctuation force we set $r_{1}=r_{2}=r$, given by the above expression, to get

$$
F=\frac{\hbar}{2 \pi c} \operatorname{Re}\left\{\int_{-\infty}^{\infty} d \omega \omega \operatorname{coth} \frac{\hbar \omega}{2 k_{B} T} \frac{\left(\frac{i m \omega}{\zeta-i m \omega}\right)^{2} e^{2 i k\left|y_{1}-y_{2}\right|}}{1-\left(\frac{i m \omega}{\zeta-i m \omega}\right)^{2} e^{2 i k\left|y_{1}-y_{2}\right|}}\right\}
$$

In the integral appearing here, the only singularities of the integrand in the upper half plane are poles of the hyperbolic cotangent at $\omega=i n \Omega, \quad n=1,2, \cdots$, where

$$
\Omega=\frac{2 \pi k_{B} T}{\hbar}
$$

is the Matsubara frequency. Therefore, we can deform the contour of integration into the upper half plane, picking up the contribution of these poles, and write

$$
F=-\frac{\hbar \Omega^{2}}{\pi c} \sum_{n=1}^{\infty} n \frac{\left(\frac{n m \Omega}{\zeta+n m \Omega}\right)^{2} e^{-2 n \Omega\left|y_{1}-y_{2}\right| / c}}{1-\left(\frac{n m \Omega}{\zeta+n m \Omega}\right)^{2} e^{-2 n \Omega\left|y_{1}-y_{2}\right| / c}}
$$

In the low temperature limit $\Omega \rightarrow 0$ and we can replace the sum by an integral to write

$$
F=-\frac{\hbar c}{\pi\left|y_{1}-y_{2}\right|^{2}} \int_{0}^{\infty} d w w \frac{\left(w e^{-w}\right)^{2}}{\left(\frac{\zeta\left|y_{1}-y_{2}\right|}{m c}+w\right)^{2}-\left(w e^{-w}\right)^{2}}
$$

This is the result for the fluctuation force at zero temperature. In the limits of large and small separation the integrals are standard, and we obtain:

$$
F=-\left\{\begin{array}{cl}
\frac{\pi \hbar c}{24\left|y_{1}-y_{2}\right|^{2}}, & \frac{\zeta\left|y_{1}-y_{2}\right|}{m c} \ll 1 \\
\frac{3 m^{2} \hbar c^{3}}{8 \pi \zeta^{2}\left|y_{1}-y_{2}\right|^{4}} & \frac{\zeta\left|y_{1}-y_{2}\right|}{m c} \gg 1
\end{array}\right.
$$

The result for small separation is the analog for our system of the London formula[9] for the force between neutral atoms, the large separation result is the analog of the CasimirPolder[10] formula.

Our aim has been to show that there is a rich variety of phenomena and plenty of unanswered questions associated with the problem of a pair of oscillators coupled to a common 
heat bath. But more we have tried to show that one must have a model of the bath for which there is a clear notion of the separation of the oscillators. For this reason we chose to discuss the simple Lamb model of a stretched string. Another obvious model would be the blackbody bath. Indeed, the Lamb model is sometimes called scalar electrodynamics and our calculation of the force between a pair of masses attached to the string is a toy example of the Lifshitz calculation of the Casimir force between two plates. Finally, we have strived to caution against a discussion of the problem based on the independent oscillator model. We have been exponents of that model because of its great generality, [1] but in its general form there is no notion of separation. The models we have advocated, the Lamb model and the blackbody bath, are special cases of independent oscillator models formulated with a clear notion of the distance between the oscillators.

The conclusion we would draw is that the problem of two or more oscillators in a common heat bath can be formulated and some of the interesting questions answered, but not from an independent oscillator model.

This work was supported in part by the National Science Foundation under grant no. ECCS-1125675.

[1] G. W. Ford, J. T. Lewis, and R. F. O'Connell, Phys. Rev. A 37 (1988) 4419.

[2] X. L. Li, G. W. Ford and R. F. O'Connell, Phys. Rev. A 41 (1990) 5287 ; ibid., Phys. Rev. E 53 (1996) 3359 .

[3] H. Lamb, Proc. London Math. Soc. 32 (1900) 208 .

[4] J. T. Lewis and L. C. Thomas, in Functional Integration, edited by A. M Arthurs, Proceedings of the International Conference on Functional Integration, Cumberland Lodge, London, 1974 (Clarendon Press, Oxford, 1975).

[5] J. J. Sakurai, Advanced quantum mechanics (Addison-Wesley, Reading, MA 1967)

[6] G. W. Ford and R. F. O'Connell, Nature, 380 (1996) 113.

[7] G. W. Ford and R. F. O'Connell, Journal of Physics A 35 (2002) 4183.

[8] G. W. Ford, J. T. Lewis and R. F. O'Connell, Phys. Rev. Lett. 55 (1985) 2273.

[9] F. London, Zeits. fur Physik 63, 245 (1926); F. London, Trans. Faraday Soc. 33, 8 (1937).

[10] H. B. G. Casimir and D. Polder, Phys. Rev. 73, 360 (1948). 